\title{
Convertible mesh dome
}

\author{
Aleksandr Ibragimov ${ }^{1, *}$, and Ljubov' Gnedina ${ }^{1}$ \\ ${ }^{1}$ Moscow State University of Civil Engineering, 129337, 26, Yaroslavskoe Shosse, Moscow, Russia
}

\begin{abstract}
The developed design of the mesh shell has the ability to fold by decreasing its dimension in one direction and increasing in the other. The fundamental difference of this design is the feasibility of folding the dome exactly wholly without the need for a complete separation of the shell into separate elements. The positive side of using such a design is expressed in the manufacture of elements, acceleration of installation and the mobility property, in which the dome can be folded and moved to a new location. The above described step-by-step description of actions in the general method of constructing the shell surface allows one to model the skeleton of a folded mesh dome. Recommendations for calculating design include a description of the methods used to perform calculations manually, the derivation of arguments in favor of computer calculation, the instructions for creating a model, the definition of rigidity and types of finite elements, as well as accounting for nonlinearity in the design of the dome in the software LIRA-CAD. The specification of the calculation scheme and the calculation itself are carried out using the general theory of shells.
\end{abstract}

\section{Introduction}

The desire of man to facilitate his work, accelerate the execution of work, as well as to reduce the cost of material resources led him along the path of inventions and discoveries. At present, a great deal of experience has been gained in designing various types of building structures, both standard and unique[1]. The idea of folding structures is far from new, but over time this topic continues to be developed and supplemented. The proof of this is the existence of a large number of registered patents.

Holding constructions have wide possibilities of practical implementation in the construction, agricultural and military sectors. Designs, as a rule, are unique and require an individual approach to their calculation and design. At the moment, a large number of possible options for folding structures of coatings have been proposed (which is taken into account in the patent databases of both Russia and the bases of other countries). It's not surprising, because positive qualities make a significant contribution to the benefit of the folding alternative compared to the stationary one. So, folding structures are indicated by their mobility. They can be brought to the construction site, laid out on site without significant labor and material resources, and at the end of their use, they can also be folded and moved to a new place.

\footnotetext{
*Corresponding author: IbragimovAM@mgsu.ru
} 
This article discusses the design of the mesh surface with a fundamentally new idea, which consist in folding the entire shell. Mesh shells have a large number of positive characteristics: light weight, high strength, survivability, the ability to cover large spans, and relatively low material costs for their production. The proposed design incorporates all the advantages of mesh shells and folding structures. Among the tradeoffs, there are high requirements for accuracy in the production of the finished shell, as well as the complexity of the design process.

\section{The method of constructing the surface of the shell (dome)}

The structural diagram is shown in Fig. 1, where: 1- the supporting element, 2-flexible cables (for the initial approximation, single lay ropes of the TK design type $1 \times 19(1+6+12)$ in accordance with GOST 3063-80 are used in the calculation. Fig.2 shows the main types of construction. All components of the structure are hinges, which is necessary to ensure the possibility of folding. To ensure durability and stability of the structure in the design position, it is necessary to introduce additional connection (light cables or ropes) that provide geometric structure for any type of load.

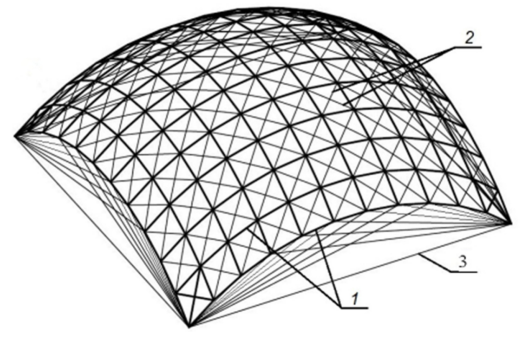

Fig.1. The structure diagram.

a)

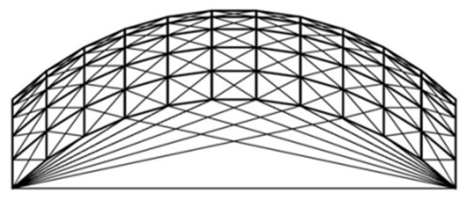

b)

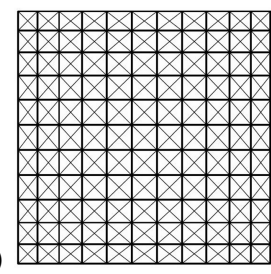

Fig.2. Views: a) front view, b) plan

From the point of view of architecture, the surface is a kind of wide-span arch -a sailing dome. The surface id built on the basis of a sphere segment with the subsequent transformations describes below.

Fig. 3 shows the surface construction scheme, where: 1 is the main circle located in the YOZ plane, 2 is the plane bounding the surface from below, 3,4,5 are construction circles having the same radius, both between themselves and with the main circle.

When constructing the surface, it is necessary to set a certain height along the $Z$ axis, that is, an arrow to raise the dome, and also know the dimensions of the object to be covered (span). Knowing these parameters, we set the main circle 1 in one of the directions (in the described case, this is the $\mathrm{Y}$ axis).

Next, select the length of one structural element (about 2-3 meters) and enter a polygon with a side of this length into the existing circle so that in the lower part of the circle on the $\mathrm{Z}$ axis lies the angle of the n-gon, and in the upper there is an orthogonal intersection of the $\mathrm{Z}$ axis and the side n-gon. This is achieved due to the odd number of sides of the n-gon. At 
this stage, it will be necessary to increase the radius of the circle 1 so that the selected polygon fits correctly. It is necessary to "multiply" the existing circle with the polygon inscribed in it in the direction of the main circle 1 along the $\mathrm{Y}$ axis with a step equal to one element of the polygon, that is, the transfer of circles is carried out at the points of the vertices of the inscribed polygon. To do this, we turn a copy of the main circle with the polygon 3 inscribed in it relative to the $\mathrm{Z}$ axis by $90^{\circ}$ and carry out the steps described above. "Reproduction" is carried out to the surface, which limits the required space, located in the positive direction of the $\mathrm{Z}$ axis in the selected coordinate system, by a circle (curve) 2. In this case, the last breeding elements are circles 5 . Next, we remove the construction lines, as well as lines that go beyond the circles 5, we leave only those parts of the polygons that are bounded by circles 5 . After that, we turn the copies of the remaining elements around the $\mathrm{Z}$ axis $90^{\circ}$ three times.

\section{Results}

As a result, we obtain the transfer surface shown in Fig.4, where: 1 is the main circle located in the $\mathrm{YOZ}$ plane, 2 is the plane bounding the surface from below, 3 is the constructed surface.

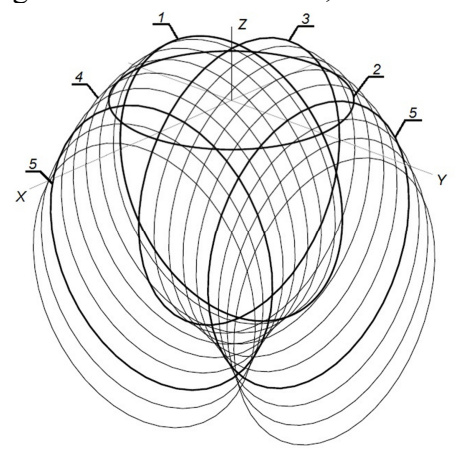

Fig.3. Scheme of surface construction.

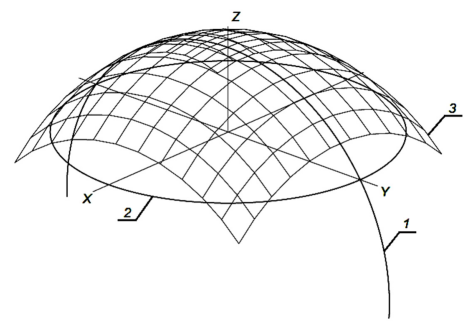

Fig. 4. The resulting surface.

\section{Conclusions}

All individual steel-bearing structural elements that make up the quadrangular cells must be of the same length. This is the main condition for the folding property of the structure. It should be noted that these constructions were performed without considering the optimization of the design; Here is a general methodology for modeling the idea of a folding mesh surface.

\section{Introduction}


Part 1 step-by-step description of the actions is given in the general methodology for constructing the shell surface, which allows us to simulate the frame of a folding mesh dome.

\section{Calculation recommendations}

There are two approaches to the manual calculation of the mesh shells [2]:

1) Discrete approach (using classical methods of structural mechanics of rod systems);

2) Replacing a discrete system with a continuum model with equivalent stiffness properties (using the theory of plates and shells).

The discrete approach is difficult to implement due to the fact that the difficulty of obtaining numerical solutions of systems of equations grows with an increase in the number of nodes and rods.

To simplify the calculation Pshenichnov G.I. based on the classical general theory of shells Vlasova V.Z. the theory of thin elastic mesh shells was created [3], which made it possible to calculate mesh shells as continuum structures with the possibility of a reverse transition from the forces of an equivalent continuous shell to the forces in individual rods.

The approaches described above require a high concentration of attention and a large amount of time in the calculation. However, at present, the level of development of computer technology allows a fairly quick calculation of complex spatial systems using settlement software systems. As an example, we give the calculation of a mesh folding surface in the LIRA-CAD software package (PK).

The design scheme was created in AutoCAD and imported into the LIRA-SAPR PK. Before starting work, the circuit is packaged; for proper packing, it is necessary to exclude conditional nodes in the crosshairs of the cables from the "stitching". In the supports, restriction of movements in all directions is specified, for the cables of the support contour it is forbidden to move along $\mathrm{Z}$ axis, in the movable nodes providing folding of the structure, hinges are set at the ends of the steel elements allowing rotation relative to the local $\mathrm{Z}$ axis. Next, the structure is divided into blocks with subsequent assignment their stiffnesses (to a first approximation) (Fig.5):

1- Steel load-bearing elements ( bent welded rectangular profile "Molodechno", C345 steel, element type- column);

2- cables of the supporting contour (KE 310 final elements, single lay rope of type TK of construction $1 \times 19(1+6+12)$ ) $\varnothing 16 \mathrm{~mm}$ according to GOST 3063-80);

3- main cables (end elements KE 310, single lay rope of TK type design 1x19( $1+6+12$ ) $\varnothing 12 \mathrm{~mm}$ according to GOST 3063-80).

Due to the introduction of hinges, the system becomes geometrical variable, therefore, to ensure rigidity and stability, ropes are added that extend along the supporting contour and separately through each cell of the sheath, which are then pulled together on one of the four structural support ( Fig.5, position 2, 3).

The authors obtained two patents for utility models [1].

In [1], a folding mash dome was patented.

Summary of utility model:

The utility model relates to the field of construction, namely to the construction of temporary structures, and is intended for use as a supporting structure of the coating, allows you to speed up the process of installation and dismantling of the structure.

A folding mesh dome consists of square and rhombic cells, the faces of which, being load-bearing structural elements, are articulated at their vertices. To ensure structural rigidity, light cables are used, fixed in cells in the form of crosshairs. Also, the cables are mounted along one supporting contour of the structure. The folding mesh dome has the ability to fold entirely along one of the main generators of the structure. 
In, an arch design was patented, which contains the upper girdle (dome element 1), puffs (dome element 3), hinges in the nodes of the dome.

Analysis of the design of the arch and dome [3,4] for progressive (avalanche-like) collapse allows us to conclude that the structure is survivable and retains its shape even if 3 puffs fail.

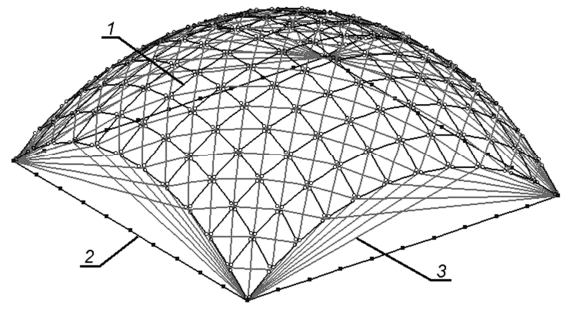

Fig. 5. Calculation scheme indicating stiffnesses, where: 1- steel load-bearing elements, 2- cables of the support loop, 3-main cables.

The design is calculated on the load from its own weight, snow and wind loads. To simplify the task of snow and wind loads, a tent cover over a dome made of technical fabric with the designated type KE 342 (geometrically non-linear universal triangular KE shell) is generally designed. Modeling of nonlinear loads is carried out using the step method provided that the step is automatically selected or geometrically and physically nonlinear problems. The calculated force combination (DCS) and the calculated load combinations ( RSN) in LIRA-CAD software are applicable only for linear calculations using the principle of superposition. When calculating taking into account nonlinearity, it is necessary to find out how the loads are interconnected over time. Thus, you need to manually select the least favorable combination of loads. The LIRA-SAPR software makes it possible to speed up this process by manually settings the required load combinations ( similar to the PCN table), which makes it possible to simulate the operation of this shell with sufficient reliability in the LIRA-SAPR PK, to obtain a calculation result for various kinds of loads and as a result select the required section of the elements.

\section{Conclusions}

Design- a folding mesh dome can be applied in various industries. For example: in agriculture -as temporary grain stores; as exhibition pavilions and spectacular facilities.

\section{References}

1. T. Bodenmueller, Streaming surface reconstruction from real time $3 D$ measurements (2009)

2. P. Beccarelli, Biax. Test. Fab. Foils. Springer, 9-33 (2015)

3. P. Beccarelli, The design, analysis and construction of tensile fabric structures (2015)

4. P. Petschek, S. Gass, Constructing Shadows: Pergolas, Pavilions, Tents, Cables, and Plants. (Walter de Gruyter 2012) 